Hautarzt 2014 · 65:168-168

DOI 10.1007/s00105-013-2661-7

Online publiziert: 16. März 2014

๑) Springer-Verlag Berlin Heidelberg 2014

J. Wohlrab ${ }^{1}$ P. Staubach ${ }^{2}$

${ }^{1}$ Universitätsklinik und Poliklinik für Dermatologie und Venerologie,

Martin-Luther-Universität Halle-Wittenberg, Halle (Saale)

${ }^{2}$ Hautklinik und Poliklinik, Universitätsmedizin Mainz, Johannes Gutenberg-Universität KöR, Mainz

\title{
Zukunft der topischen Therapie
}

Die professionelle topische Therapie zählt zu den Kernkompetenzen der Dermatologen. Im Behandlungsalltag ist die epikutane Applikation von Wirksubstanzen die mit Abstand am häufigsten genutzte Therapieform. Vor diesem Hintergrund ist die fundierte und stetige inhaltliche Auseinandersetzung in Weiter- und Fortbildung zwingend notwendig, um Qualitätsstandards zu erhalten und auszubauen. In den letzten Jahren standen die Systemtherapien besonders im fachlichen Fokus, so dass die topischen Therapieoptionen etwas aus unserem Blick geraten sind. Dennoch haben sich auch hier neue Möglichkeiten und Entwicklungen ergeben, die bemerkenswert sind. Zudem bietet die Magistralrezeptur nicht nur die Möglichkeit, das Spektrum der verfügbaren Spezialitäten zu ergänzen bzw. zu erweitern, sondern auch seltene therapeutische Situationen abzudecken. Wichtig in diesem Zusammenhang ist die Kenntnis neuer Standards, die sich durch die Novellierung der Apothekenbetriebsordnung ergeben, welche dem Apotheker eine Plausibilitätstestung und die Dokumentationspflicht von Rezepturen auferlegt. Diese Bedingungen führen dazu, dass galenisch unsichere oder gar instabile Rezepturen bzw. Rezepturen mit nicht arzneibuchkonformen Inhaltstoffen (z. B. die meisten Kosmetika) nicht mehr hergestellt werden dürfen.

Der professionelle Einsatz topischer Therapie muss erlernt werden und ist in vielen Aspekten nicht trivial. Das Einbeziehen von krankheitsphasen-, lokalisations- und altersspezifischen Aspekten in therapeutische Entscheidungsprozes- se bedarf klinischer Erfahrung und eines fundierten Wissens zu indikationsbezogenen pathogenetischen Abläufen im Hautorgan. Dies muss gepaart sein mit einem galenischen Grundverständnis, Kenntnissen zum molekularen Aufbau der Haut, insbesondere des Stratum corneum, und pharmakologischem Wissen zu Penetrationskinetik sowie der Eigenwirkung von Vehikelsystemen. All dies schafft die Grundlage für eine evidenzbasierte, professionelle Anwendung topischer Präparate für die Prophylaxe und Therapie in unterschiedlichen klinischen Behandlungssituationen.

Im vorliegenden Leitthemenheft haben sich engagierte und erfahrene Fachkolleginnen und -kollegen in klar gegliederten Beiträgen der Darstellung der genannten Zusammenhänge gewidmet. Ausgehend von den morphologischen, funktionellen und pharmakologischen Grundlagen, den regulatorischen Erfordernissen, dem professionellen Einsatz von Magistralrezepturen sowie den alters- und lokalisationsspezifischen Besonderheiten, nehmen die Autoren zu den Standards der Verwendung von Topika in definierten klinischen Behandlungssituationen Stellung. Zudem werden Hinweise für die Therapieplanung formuliert. Dieser inhaltliche Duktus zielt auf den praktischen Nutzen für den Leser im täglichen Behandlungsalltag und soll Ihnen Hinweise, Unterstützung und Anregung geben.

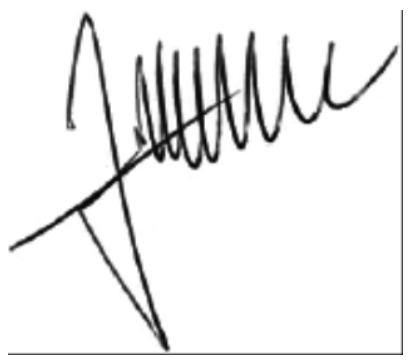

Johannes Wohlrab

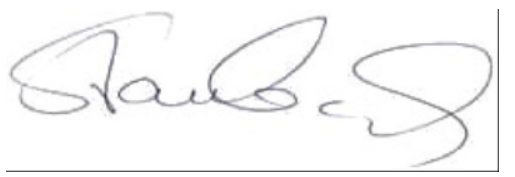

Petra Staubach

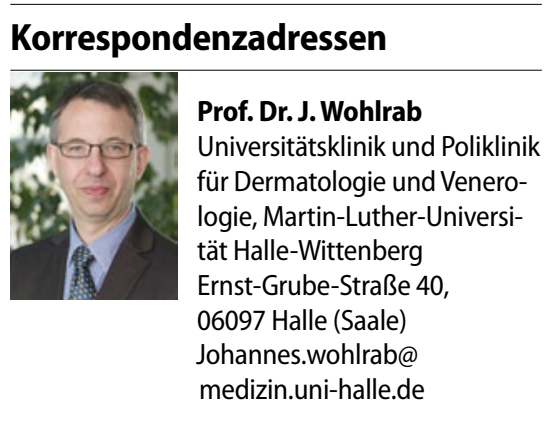

PD Dr. P. Staubach
Hautklinik und Poliklinik,
Universitätsmedizin Mainz,
Johannes Gutenberg-
Universität KöR
Langenbeckstr. 1,55131 Mainz
petra.staubach@
unimedizin-mainz.de

Interessenkonflikt. J. Wohlrab und P. Staubach geben an, dass kein Interessenkonflikt besteht. 